\title{
The Validity of the Invasive Tests for Helicobacter Pylori Diagnosis is Unequally Affected by the Consumption of Antibiotics or Pump Inhibitors. Test Performance under Real-World Conditions
}

\author{
Javier Alcedo ${ }^{1,2}$, Diego Casas ${ }^{1,2}$, Jesús Gotor ${ }^{1,2}$, Miguel Lafuente ${ }^{3}$, Mónica Llorente ${ }^{1}$, Patricia Sanz-Segura ${ }^{1,2}$, Rosario \\ Monzón ${ }^{1}$, Carlos Hörndler ${ }^{4}$, Edgar Peña-Galo²
}

1) Department of Digestive

Diseases. Miguel Servet

University Hospital. Zaragoza;

2) Aragón Health Research

Institute (IIS Aragón);

3) Department of Statistical

Methods. University of

Zaragoza. Zaragoza;

4) Department of Pathology.

Miguel Servet University

Hospital. Zaragoza, Spain.

\section{Address for correspondence: \\ Javier Alcedo \\ Department of Digestive \\ Diseases, Miguel Servet \\ University Hospital, Aragón \\ Health Research Institute (IIS \\ Aragón) Zaragoza. Spain. \\ ORCID ID: https://orcid. \\ org/0000-0001-6522-6682. \\ jalcedo@telefonica.net}

\section{ABSTRACT}

Background \& Aims: Patients undergoing upper endoscopy have often used proton pump inhibitors (PPI) and/or antibiotics $(\mathrm{ABx})$ recently. Both drugs have been associated with a poorer yield of the Helicobacter pylori (H. pylori) diagnostic tests. The aim was to assess the accuracy of the polymerase chain reaction test (qPCR), histological exam (HE) and ultra-fast urease test (UFUT) for $H$. pylori detection in patients that recently used PPI or ABx.

Methods: Prospective study recruiting 206 patients who underwent upper endoscopy and gastric biopsies. Demographics and use of PPI/ABx were obtained. Sensibility (Sn), specificity (Sp), predictive value (PV), likelihood ratio (LR) and PABAK concordance index, were calculated, considering as the gold standard the positivity of 2 out of 3 analyzed tests. A global analysis and another one based on the PPI/ABx intake were performed.

Results: $48.5 \%$ of patients used PPI and $12.8 \%$ ABx within the 2 and 4 weeks prior to endoscopy, respectively. The UFUT was positive in $13.1 \%$ of patients, HE in $34 \%$ and qPCR in $35.9 \%$. UFUT achieved lower Sn (37\%) than HE $(98 \%)$ and qPCR $(98 \%)(\mathrm{p}<0.001)$ overall. ABx were associated with lower Sn in HE $(\mathrm{p}=0.04)$ and lower $\mathrm{Sp}$ in $\mathrm{qPCR}(\mathrm{p}=0.03)$. PPI did not associate with a significant drop in $\mathrm{Sn}$ and $\mathrm{Sp}$. The concordance between HE and qPCR was 0.83 (95\%CI: 0.73-0.89).

Conclusions: Under real world conditions, the accuracy and concordance of HE and qPCR to diagnose $H$. pylori were excellent, but UFUT achieved unsatisfactory outcomes. The intake of ABx was associated with the worse performance, fundamentally for HE. The PPI did not reduce the tests' yield significantly.

Key words: Helicobacter pylori - qPCR - ultra-fast urease test - histological exam - proton pump inhibitor - antibiotic.

Abbreviations: ABx: antibiotic; H. pylori: Helicobacter pylori; HE: histological exam; NLR: negative likelihood ratio; NPV: negative predictive value; PLR: positive likelihood ratio; PPI: proton pump inhibitor; PPV: positive predictive value; qPCR: real-time quantitative polymerase chain reaction test; RUT: rapid urease test; UFUT: ultra-fast urease test.

\section{INTRODUCTION}

Helicobacter pylori (H. pylori) is a gram-negative bacillus able to colonize the gastroduodenal mucosa prompting inflammation and immune response, and it modulates the local $\mathrm{pH}$ by means of urea hydrolysis $[1,2]$. These pathogenic characteristics are used for most of the tests to make the infection diagnosis. Furthermore, the enzyme that unfolds the urea is composed of two structural subunits, ureA and $u r e B$, which are codified by target genes for the molecular detection of $H$. pylori [3-6]. Nowadays, multiple techniques for $H$. pylori infection diagnosis are available. Depending on if the performance of an endoscopy is needed or not, they are classified as invasive or non-invasive tests [7-11].

The faster, cheaper and easier to perform invasive method is the rapid urease test (RUT). When the test is carried out under optimal conditions the sensitivity and specificity values are close to $80-100 \%$ and $95 \%$, respectively. Its accuracy diminishes in the presence of blood in the stomach and due to proton pump inhibitor $(\mathrm{PPI})$ or antibiotic $(\mathrm{ABx})$ consumption. A minimum of $10^{5}$ microorganisms per sample is required to gain a positive result $[12,13]$. In the histological exam (HE) the presence of typical bacteria in an inflamed tissue area is considered diagnostic of a $H$. pylori infection. Its sensitivity and 
specificity are estimated at around $94 \%$ and $99 \%$, respectively [14], although it can change depending on the biopsied gastric area, the distribution and density of the pathogen, the pathologist experience and the chosen stain. Additionally, it could be influenced by drug consumption (PPI and ABx), and it is not able to diagnose metabolically inactive forms. The culture of samples obtained by gastric biopsy shows specificity of $100 \%$, but sensitivity close to $80 \%$, so it is not recommended as a first choice diagnostic option. Finally, in some research the molecular techniques based on real-time quantitative polymerase chain reaction (qPCR), using gastric biopsies, have demonstrated sensitivity and specificity over RUT, HE and culture. qPCR is not influenced by blood presence in the stomach, and it is able to detect both $H$. pylori forms, helical and coccoid, even with low bacterial density [9-11]. It also allows detecting virulence and antibiotic-resistance specific genes. PCR techniques in samples from the oral cavity or stool have been recently developed, and some novelties such as the detection of $16 \mathrm{~S}$ rRNA and ure $C$ genes, combinations with ureA, and multigenic tests, could increase the method validity [15].

In spite of successive technical improvements, a gold standard for the H. pylori diagnosis has not yet been defined. The qPCR has shown advantages regarding other choices when the test is carried out during a peptic ulcer bleeding [16]. Nevertheless, its use has not been added to the diagnostic routine in a widespread way, and the potential advantage regarding other methods in non-urgent real-world conditions is unknown. Under these conditions those patients who have not stopped the PPI or ABx consumption during the required time are often required to be tested, or simply patients where the recent intake of any of these drugs is unknown. In this scenario the alternative tests significantly decrease its sensitivity [17], and qPCR is expected to be the gold standard. Therefore, the aim of this study was to determine and compare the accuracy of UFUT, HE and qPCR in the H. pylori infection diagnosis under real world conditions, assessing the burden of PPI and $\mathrm{ABx}$ intake in the outcomes. The influence of clarithromycin resistance on test yield was also studied.

\section{METHODS}

A prospective, single center research was performed reflecting real world conditions, following the current guidelines. Adults older than 18 years, in whom $H$. pylori infection test and upper endoscopy were indicated, were consecutively recruited. The exclusion criteria were known and untreated $H$. pylori infection, prior successful eradication treatment, partial gastrectomy, active upper gastrointestinal bleeding, pregnancy or breastfeeding, severe comorbidity (groups IV and V of the American Society of Anesthesiologists -ASA- classification). H. pylori infection was considered as proven (gold standard) when at least two of the three diagnostic tests described below were positive.

Biopsy samples from the greater curvature of the antrum and incisura angularis were obtained during the upper endoscopy for ultra-fast urease test. The Biohit Ultra-Fast UFT300 Helicobacter pylori Quick Test ${ }^{\oplus}$ (Biohit HealthCare ltd, Cheshire, United Kingdom) was used, evaluating the results after 5 minutes of the incubation period, according to the manufacturer's instructions.

Three biopsy samples from incisura angularis and greater curvature of antrum and corpus were obtained for HE. Afterwards, the paraffin-embedded tissue sections were stained with hematoxylin and eosin and studied for an expert pathologist blind to the other test's results.

The samples used for UFT were immediately stored at $-20^{\circ} \mathrm{C}$ and further processed for the qPCR test (no later than 2 weeks). Viasure Helicobacter pylori + Clarithromycin resistance Real Time PCR Detection Kit (CerTest Biotec, S.L. Zaragoza, Spain) was employed. After isolation of DNA with the Viasure RNADNA Extraction kit (CerTest Biotec, S.L. Zaragoza, Spain), the identification of $H$. pylori, clarithromycin resistance and/ or clarithromycin wild-type sequence in the $23 \mathrm{~S}$ rRNA was performed by the amplification of a conserved region of the ure $B$ and $23 \mathrm{~S}$ rRNA genes respectively, using specific primers and a fluorescent-labeled probe. Point mutations in the $23 \mathrm{~S}$ rRNA gene (A2142G and A2143G), which confer resistance to Clarithromycin were amplified and detected in the FAM channel; $H$. pylori DNA targets were amplified and detected in the ROX channel; clarithromycin wild-type sequence in the $23 \mathrm{~S}$ rRNA gene DNA targets were amplified and detected in HEX channel and the internal control (IC) in the Cy5 channel. The fluorescence was measured on the Real Time PCR platform (Bio-Rad CFX96 ${ }^{\text {Ts }}$ Real-Time PCR Detection System).

A positive and a negative control were included in every run to validate the reaction, as well as an inner control to verify the correct working of the amplification mixture. The test results were understood as is shown in Table I. The sample was considered positive if amplification was observed before 40th cycle, or when a starting high number of the target nucleic acid copies were presented. The result was invalidated, and the assay was repeated when an amplification graphic was observed for the negative control or the signal was lacking in positive control. If the inner control signal was lacking the assay was also repeated but diluting the sample 1:10, or the extraction was done again to rule out possible inhibition problems.

Table I. qPCR test results interpretation

\begin{tabular}{ccccc}
\hline Sample & $\begin{array}{c}\text { Inner } \\
\text { control }\end{array}$ & $\begin{array}{c}\text { Negative } \\
\text { control }\end{array}$ & $\begin{array}{c}\text { Positive } \\
\text { control }\end{array}$ & Interpretation \\
\hline+ & $+/-$ & - & + & H. pylori Positive \\
- & + & - & + & H. pylori Negative \\
- & - & - & - & No valid \\
+ & + & + & + & No valid \\
\hline
\end{tabular}

qPCR: real-time quantitative polymerase chain reaction

To calculate the sample size (n), an H. pylori prevalence of $75 \%$ in patients with indication of upper endoscopy was assumed, such as has been reported for dyspeptic patients [14], an UFUT sensibility under PPI consumption not over $80 \%$ and $85 \%$ for all cases, just as $95 \%$ for qPCR under similar conditions, based on disposable data $[16,17]$. The 0.9 confidence level and a standard error of 0.05 to estimate sensitivity were used. With these data the calculated n was 203 patients who underwent endoscopy. 
Variables of gender, age, endoscopy indication, prior PPI and $A B x$ intake (last 2 and 4 weeks, respectively), regular alcohol consumption and smoking habit, as well as $H$. pylori tests, endoscopy and histological exam results, were collected. Quantitative variables were expressed as means and standard deviation and categorical ones as a proportion with $95 \%$ confidence interval (CI). A descriptive study was carried out, and median or Barnard's test were used in order to assess the independence of the variables. Ratios of sensitivity, specificity, positive and negative predictive values (PPV and NPV), and positive and negative likelihood ratios (PLR and NLR) for each test, were calculated. CI for predictive values were established by means of asymptotic standard logit intervals, using the adjusted method in the presence of null values. An analytical research assessing the $\mathrm{Sn}$ and $\mathrm{Sp}$ achieved for each test was performed, and McNemar's or Barnard's test were used, according to matching. A prevalence-adjusted and bias-adjusted kappa coefficient (PABAK) was used to assess the agreement between diagnostic tests. The p-value less than 0.05 was considered significant.

Written informed consent for participation was obtained from each of the patients before entry into the study. The patient information was managed according to Spanish Constitutional Law 15/1999 about Personal Data Protection. The study protocol conforms to the ethical guidelines of the 1975 Declaration of Helsinki and was approved by the Ethical
Committee of the Aragon Institute of Health Science (CP-CI PI16/0234).

\section{RESULTS}

Two hundred and six patients were recruited, comprising 142 females (68.93\%) and 64 males. The average was 53.2 years (range: 19-86). Fifty-eight (28.2\%) were current smokers, while $14(6.8 \%)$ regularly (daily) consumed alcohol. The endoscopies were indicated for dyspeptic symptoms in $72.8 \%$ and the result was mostly normal in $55.8 \%$. No association was found among the distribution of these variables and the outcomes of the evaluated diagnosis test (Table II).

In the two weeks before endoscopy 99 patients had consumed PPI (48.53\% out of 204 patients with known data about this consumption), 67 of them in daily intake (32.84\%); while in prior 4 weeks 26 patients had consumed $\mathrm{ABx}(12.81 \%$ out of 203 with known data). In all patients UFUT was positive in 27 (13.11\%), HE in 70 (33.98\%) and qPCR in 74 (35.92\%). Among PPI consumers UFUT was positive in 10 patients (10.1\%), HE in 29 (29.29\%) and qPCR in 32 (32.32\%). Among ABx consumers UFUT was positive in 3 (11.54\%), HE in 7 (26.92\%), and qPCR in 10 (38.46\%). Among both of them, PPI or ABx consumers, the positive numbers were $10.28 \%$ for UFUT, $28.97 \%$ for $\mathrm{HE}$ and $33.64 \%$ for qPCR.

Table II. Patients' demographics and endoscopy findings compared with test results

\begin{tabular}{|c|c|c|c|c|c|}
\hline \multirow[t]{4}{*}{ Gender } & & Female & \multicolumn{2}{|l|}{ Male } & p-value \\
\hline & UFUT + & $10.94 \%$ & \multicolumn{2}{|l|}{$14.08 \%$} & 0.602 \\
\hline & $\mathrm{HE}+$ & $35.92 \%$ & \multicolumn{2}{|l|}{$29.69 \%$} & 0.419 \\
\hline & $\mathrm{qPCR}+$ & $38.73 \%$ & \multicolumn{2}{|l|}{$29.69 \%$} & 0.237 \\
\hline \multirow[t]{4}{*}{ Age (years): mean (SD) } & & Positive & \multicolumn{2}{|l|}{ Negative } & p-value \\
\hline & UFUT & $52.5(14.5)$ & \multicolumn{2}{|l|}{$53.3(16.4)$} & 0.199 \\
\hline & $\mathrm{HE}$ & $53.3(14.5)$ & \multicolumn{2}{|l|}{$53.1(16.9)$} & 0.995 \\
\hline & qPCR & $54.2(13.2)$ & \multicolumn{2}{|l|}{$52.6(17.5)$} & 0.982 \\
\hline \multirow[t]{4}{*}{ Smoking habits } & & Yes & \multicolumn{2}{|l|}{ No } & $\mathrm{p}$-value \\
\hline & UFUT + & $12.07 \%$ & \multicolumn{2}{|l|}{$13.79 \%$} & 0.755 \\
\hline & $\mathrm{HE}+$ & $24.14 \%$ & $38.62 \%$ & & 0.051 \\
\hline & qPCR + & $32.76 \%$ & $37.93 \%$ & & 0.497 \\
\hline Regular alcohol consumption & & Yes & No & & p-value \\
\hline & $\mathrm{UFUT}+$ & $7.14 \%$ & $13.76 \%$ & & 0.619 \\
\hline & $\mathrm{HE}+$ & $28.57 \%$ & $34.92 \%$ & & 0.872 \\
\hline & $\mathrm{qPCR}+$ & $21.43 \%$ & $37.57 \%$ & & 0.265 \\
\hline Main indication for endoscopy & & Dyspepsia & Others & & p-value \\
\hline & UFUT + & $12.67 \%$ & $14.29 \%$ & & 0.802 \\
\hline & $\mathrm{HE}+$ & $34,00 \%$ & $33.92 \%$ & & 0.999 \\
\hline & $\mathrm{qPCR}+$ & $34,00 \%$ & $41.07 \%$ & & 0.402 \\
\hline Main endoscopy findings & & Normal & $\begin{array}{l}\text { ic gastro- } \\
\text { disease }\end{array}$ & Others & $\mathrm{p}$-value \\
\hline & UFUT + & $12.17 \%$ & & $8.70 \%$ & 0.658 \\
\hline & $\mathrm{HE}+$ & $33.91 \%$ & & $30.43 \%$ & 0.910 \\
\hline & $\mathrm{qPCR}+$ & $35.65 \%$ & & $26.09 \%$ & 0.499 \\
\hline
\end{tabular}

HE: histological exam; qPCR: real-time quantitative polymerase chain reaction; SD: standard deviation; UFUT: ultra-fast urease test. Statistics: median and Barnard's test. 
The estimated test values of sensitivity, specificity, predictive values and likelihood ratios are shown in Table III. UFUT globally achieved lower sensitivity than HE and qPCR $(\mathrm{p}<0.001)$. The $\mathrm{ABx}$ intake was associated with lower sensitivity in HE ( $\mathrm{p}=0.04)$, and lower specificity in $\mathrm{qPCR}$ $(p=0.03)$ in comparison with non-drug intake. The PPI consumption was not associated with a statistically significant lowering of the tests' accuracy. Nevertheless, there was a trend to lower sensitivity of the qPCR in patients with daily intake regarding those with occasional or no PPI-intake $(\mathrm{p}=0.06)$, and also regarding no PPI intake $(\mathrm{p}=0.07)$ (Fig. 1). The PABAK concordance index between results of $\mathrm{HE}$ and qPCR was 0.83 (95\%CI: 0.73-0.89), with McNemar mid-p-value of 0.36 , meaning an almost perfect agreement between both test results. The same index was 0.47 (95\%CI: 0.33-0.58) between UFUT and qPCR, and 0.50 (95\%CI: 0.38-0.62) between UFUT and HE, both with McNemar $<0.001$, showing significant disagreement among UFUT and the other two tests.

The clarithromycin resistance presence in the $H$. pylori positive samples was detected in 16 out of 74 patients with positive qPCR (21.62\%). Among the patients with resistance, 5 were positive in UFUT and 14 in HE. Among the 58 patients without resistance 18 were positive in UFUT and 49 in HE. The ratio resistant/sensitive of UFUT and HE were separately homogeneous ( $\mathrm{p}=1$ and $\mathrm{p}=0.922$, respectively), meaning that both tests' sensitivity was not modified according to clarithromycin resistance. As in the overall analysis, a lowering in the UFUT sensitivity regarding HE one was seen in both groups, with and without clarithromycin resistance $(\mathrm{p}=0.002$ and $\mathrm{p}<0.001$, respectively); so that we can conclude that $\mathrm{HE}$ reached a better diagnostic performance than UFUT regardless of the resistance status.

\section{DISCUSSION}

This is a prospective study that compares the accuracy of the two most used invasive $H$. pylori diagnostic tests and
qPCR, in real world conditions. The included patients were candidates for upper endoscopy and the $H$. pylori investigation was indicated for them. In this scenario more than $48.5 \%$ consumed PPI (almost 33\% in a daily regimen) and $12.81 \%$ had taken some dose of antibiotic in the 2 and 4 weeks prior to endoscopy, respectively. These percentages are very similar to the $47 \%$ of patients taking PPI reported by El-Zimaity el al. [18], but lower than $61 \%$ in the study of Shirin el al. [19] also in patients referred for upper endoscopy. In the latter only $4.5 \%$ of patients stopped PPI 2 weeks before the procedure and $10.8 \%$ had been on antibiotic treatment within the past four weeks. These data suggest that in $H$. pylori diagnosis by means of invasive tests, around half of the time the ideal conditions are not kept to, which could affect the accuracy of the method.

The current Clinical Guidelines [7, 8] state that PPI should be discontinued at least 2 weeks before testing for $H$. pylori infection, while antibiotics and bismuth compounds should be discontinued at least 4 weeks before the test. In vitro studies found that high doses of PPI have negative effects on H. pylori viability, inhibiting the growth of isolates and urease activity, with bacterial morphology changing from spiral to coccoid $[17,20]$. In parallel, the authors reported that in their series the PPI consumption was significantly higher in RUT-negative patients than in positives. Other researchers concluded that the consumption of both, $\mathrm{ABx}$ and PPI, could lead to reduction in the antrum of the $H$. pylori population $[21,22]$. This phenomenon has been explained by changes in the gastric surface that could occult the bacteria, elevation of the intragastric $\mathrm{pH}$ making an unfavorable environment for H. pylori to develop, and the antimicrobial effect, not only of antibiotics but also of PPI. All of these circumstances could lead to false-negative results especially of RUT and HE.

The level of concordance among the tests according to the results of the calculated PABAK coefficient must be considered very good between $\mathrm{HE}$ and qPCR, but only mild/moderate between UFUT and qPCR, with significant disagreement between the latter two. In fact, we found a pronounced drop in

Table III. Comparison of the tests' performance in different scenarios

\begin{tabular}{|c|c|c|c|c|c|c|c|}
\hline & & Sn $(95 \%$ CI $)$ & $\mathrm{Sp}(95 \% \mathrm{CI})$ & PPV (95\% CI) & NPV (95\% CI) & PLR $(95 \%$ CI $)$ & NLR (95\% CI) \\
\hline \multirow[t]{3}{*}{ Overall } & UFUT & $0.37^{\star *}(0.25-0.49)$ & $0.98(0.94-1)$ & $0.89(0.71-0.96)$ & $0.77(0.74-0.80)$ & $17.35(5.42-55.56)$ & $0.64(0.53-0.78)$ \\
\hline & $\mathrm{HE}$ & $0.98(0,92-1)$ & $0.96(0.91-0.98)$ & $0.91(0.83-0.96)$ & $0.99(0.95-1)$ & $\begin{array}{l}23.14(10.57- \\
50.65)\end{array}$ & $0.02(0.00-0.11)$ \\
\hline & $\mathrm{qPCR}$ & $0.98(0,92-1)$ & $0.93(0.87-0.97)$ & $0.86(0.78-0.92)$ & $0.99(0.95-1)$ & $13.88(7.63-25.25)$ & $0.02(0.00-0.12)$ \\
\hline \multirow[t]{3}{*}{ With Antibiotics } & UFUT & $0.6(0.15-0.95)$ & $1(0.84-1)$ & $0.70(0.28-0.88)$ & $0.90(0.81-0.95)$ & $\operatorname{Inf}(\mathrm{NaN}-\operatorname{Inf})$ & $0.40(0.14-1.17)$ \\
\hline & $\mathrm{HE}$ & $0.8 \dagger(0.28-0.99)$ & $0.86(0.64-0.97)$ & $0.57(0.30-0.81)$ & $0.95(0.76-0.99)$ & $5.60(1.80-17.43)$ & $0.23(0.04-1.36)$ \\
\hline & $\mathrm{qPCR}$ & $1(0.48-1)$ & $0.76^{\dagger}(0.53-0.92)$ & $0.42(0.25-0.58)$ & $0.95(0.80-0.98)$ & $4.20(1.95-9.03)$ & $0.00(0.00-\mathrm{NaN})$ \\
\hline \multirow[t]{3}{*}{ With PPI (any intake) } & UFUT & $0.33^{* \star}(0.16-0.55)$ & $0.97(0.91-1)$ & $0.80(0.48-0.95)$ & $0.82(0.77-0.86)$ & $12.50(2.85-54.90)$ & $0.68(0.51-0.91)$ \\
\hline & $\mathrm{HE}$ & $1(0.86-1)$ & $0.93(0.85-0.98)$ & $0.79(0.62-0.87)$ & $0.98(0.91-0.99)$ & $15.00(6.43-18.99)$ & $0.00(0.00-\mathrm{NaN})$ \\
\hline & qPCR & $1(0.86-1)$ & $0.89(0.80-0.95)$ & $0.72(0.57-0.81)$ & $0.98(0.91-0.99)$ & $9.38(4.87-18.05)$ & $0.00(0.00-\mathrm{NaN})$ \\
\hline \multirow[t]{3}{*}{ Daily PPI intake } & UFUT & $0.35^{\star *}(0.14-0.62)$ & $0.96(0.86-1)$ & $0.75(0.40-0.93)$ & $0.81(0.75-0.86)$ & $8.82(1.96-39.65)$ & $0.67(0.47-0.96)$ \\
\hline & $\mathrm{HE}$ & $1(0.80-1)$ & $0.94(0.83-0.99)$ & $0.79(0.59-0.89)$ & $0.98(0.88-0.99)$ & $16.67(5.56-49.93)$ & $0.00(0.00-\mathrm{NaN})$ \\
\hline & qPCR & $1(0.80-1)$ & $0.86(0.73-0.94)$ & $0.67(0.50-0.78)$ & $0.97(0.87-0.99)$ & $7.14(3.59-14.20)$ & $0.00(0.00-\mathrm{NaN})$ \\
\hline
\end{tabular}

HE: histological exam; Inf: Infinite; NaN: Not a number (impossible-to-calculate value); NLR: negative likelihood ratio; NPV: negative predictive value; PLR: positive likelihood ratio; PPI: Proton pump inhibitor; PPV: positive predictive value; qPCR: real-time quantitative polymerase chain reaction; Sn: sensibility; Sp: Specificity. UFUT: ultra-fast urease test; ${ }^{* *} \mathrm{p}<0.001$ regarding other test results; ${ }^{\dagger} \mathrm{p}<0.05$ regarding non-drug intake. Statistics: McNemar's and Barnard's tests. 


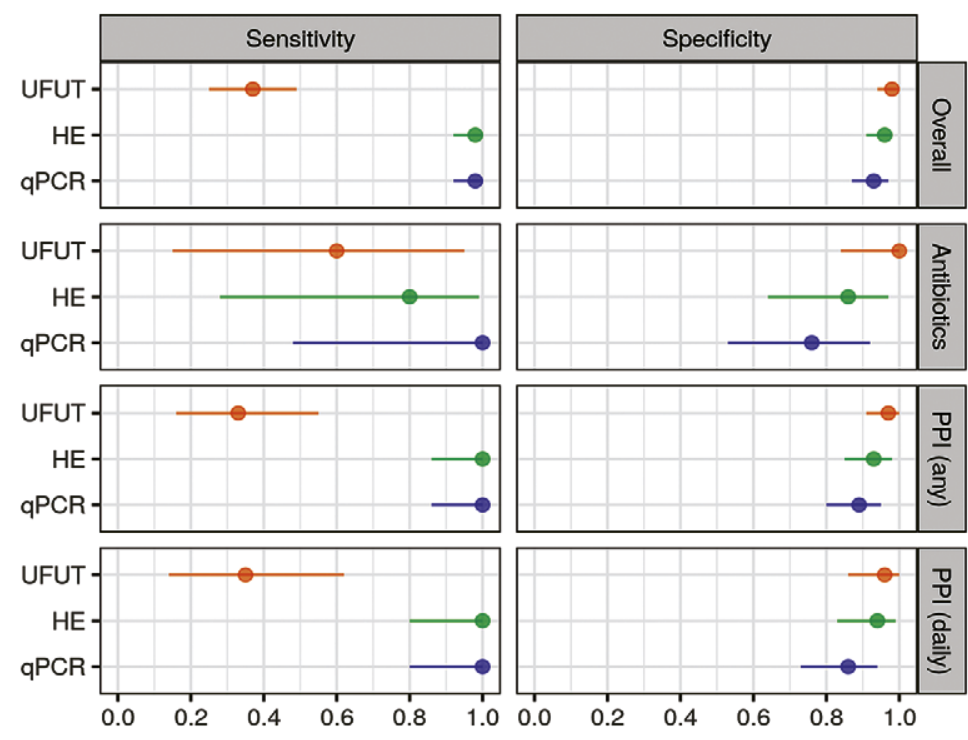

Fig. 1. Sensitivity and specificity (mean and 95\% CI) achieved for each test in different scenarios. PPI: Proton pump inhibitor. For abbreviations see Table II.

the sensitivity of UFUT. Our results are consistent with those reported by Siavoshi et al. [17], which showed a lowering of sensitivity from $92.2 \%$ up to $74.4 \%$ in patients without vs. with PPI consumption, respectively. Other researchers using different RUT devices described a sensitivity ratio drop from $71.9 \%$ (without PPI) to $43.3 \%$ (with PPI) [23].

The RUT chosen in our study was the UFUT, sampling antrum and incisura angularis, and with reading after 5 minutes, following the manufacturer's instructions. In patients not selected by PPI consumption, the UFUT with reading at 30 and 60 minutes have demonstrated to be at least as accurate as conventional RUT with reading up to 24 hours [24]. Previously, Vaira et al. [25] did not find differences between UFUT with reading at 60 and 5 minutes in specificity (100\% in both) and sensitivity [96.2 (95\%CI: 94.5-98) vs. 94.5 (95\%CI: 92.4-96.6)], in this case for patients without prior PPI.

Regarding the HE test, in our series the PPI intake was not associated with a lowering in accuracy, nevertheless the antibiotic consumption decreased both sensitivity and specificity. Bazin et al. [26] also reported non-significantstatistical differences in the sensitivity of the HE, in 192 patients H. pylori positive, 52 of them taking PPI. Other authors have informed fall in sensitivity of HE taking PPI, in both antrum and corpus biopsies, using PCR as gold standard, but the sample size analyzed was small and their ratios were even lower than others reported in earlier studies for the same researchers. Furthermore, they yield very low specificity of HE and PCR regarding that commonly reported, which was not explained $[27,28]$. On the other hand, classical studies also found a drop in the sensitivity of $\mathrm{HE}$ and culture after taking $\mathrm{ABx}$, which explains the guidelines' recommendations concerning stopping them 4 weeks before diagnostic tests [8].

The exposed tests' limitations could be saved, partially at least, for the molecular techniques since they have gene targets not affected by bacterial morphology and they are able to detect smaller bacterial load. In fact, the molecular diagnosis techniques have been demonstrated to be less influenced by PPI consumption and to detect additional $H$. pylori positive cases $[29,30]$ which is in accordance with our results (trend to lower sensitivity among PPI consumers but not achieving statistical significance). Additionally, a very high specificity of the $23 \mathrm{~S}$ qPCR, close to $100 \%$, has been already established [26]. For all these reasons, qPCR has been proposed as an additional test to assure the diagnosis in RUT negative patients, while it also can provide determining information for the treatment choice, based on the ABx-resistance. In the current research the clarithromycin-resistance ratio was $21.6 \%$, which is close to the almost $18 \%$ recently reported from a neighboring geographical area [31], and this condition did not affect the test yield.

In spite of the points made above, a limitation of our study might be that UFUT test might not be representative of all the remaining RUT tests, and there is scarce prior research about this issue. As shown below, Vaira et al. [25] did not find differences reading test results in 5 or in 60 minutes. McNichols et al. [24] reported higher frequency of positive tests in a prospective series in cases read at 60 minutes regarding those read at 5 minutes ( $90 \%$ vs $78 \%$ ), but these results came from different populations and settings, which do not allow their comparison. Along these lines we studied in our series the positive UFUT test read just before qPCR processing (between 24 hours to 2 weeks after biopsy), named as late UFUT (L-UFUT). We found higher sensitivity ratio than conventional UFUT $(0.77$ vs 0,37$)$, without loss of specificity and with a moderate correlation phi coefficient $(0.60)$ between both, UFUT and L-UFUT, suggesting that a later reading of UFUT test could improve the yield of the test. However, the sensitivity of L-UFUT measure was also significantly lower than the same of $\mathrm{HE}$ and qPCR in practically all the scenarios (data in Supplementary file). Therefore, the choice of different RUT techniques does not seem to substantially modify the main results.

\section{CONCLUSIONS}

Under real world conditions a large number of patients who underwent upper endoscopy did not stop the PPI and/or $\mathrm{ABx}$ consumption for the period of time recommended by the 
current Clinical Guidelines to ideally investigate the $H$. pylori infection. In this scenario the UFUT test has shown lower sensitivity than HE and qPCR. The last two tests achieved on the whole sample high accuracy and high level of agreement. The intake of PPI did not significantly affect the accuracy of $\mathrm{qPCR}$ and HE, whereas the intake of $\mathrm{ABx}$ decreased the sensitivity of HE. The resistance to the clarithromycin factor did not have a relevant burden on the test yield. So, in view of these data the isolated use of UFUT should be strongly advised against in situations where conditions are far from ideal. The qPCR could be the best choice among the invasive tests in real practice, used as a sole method or as additional test in UFUT negative cases.

Conflicts of interest: None to declare.

Authors' contributions: J.A. planned and coordinated the study, analyzed the data and wrote the manuscript drafts. D.C., J.G. and E.P.G. designed and programmed the electronic case report form and acquired data. M.L.L., P.S.S. and R.M. acquired data. M.L. performed the statistical analysis. C.H. performed the histological exams. D.C., J.G., E.P.G., M.L., C.H., M.L.L., P.S.S. and R.M. critically reviewed the manuscript draft. All the authors approved the final version of the manuscript.

Acknowledgements: We acknowledge the technical support of Concepción Gil, Henar Alonso and CERTEST-Biotec in the carrying out of the qPCR test and the interpretation of its results.

Supplementary material: To access the supplementary material visit the online version of the J Gastrointestin Liver Dis at http://dx.doi. org/10.15403/jgld-3161.

\section{REFERENCES}

1. Suerbaum S, Michetti P. Helicobacter pylori infection. N Engl J Med 2002;347:1175-1186. doi:10.1056/NEJMra020542

2. Wang F, Meng W, Wang B, Qiao L. Helicobacter pylori-induced gastric inflammation and gastric cancer. Cancer Lett 2014;345:196-202. doi:10.1016/j.canlet.2013.08.016

3. Kusters JG, van Vliet AH, Kuipers EJ. Pathogenesis of Helicobacter pylori infection. Clin Microbiol Rev 2006;19:449-490. doi:10.1128/ CMR.00054-05

4. Schabereiter-Gurtner C, Hirschl AM, Dragosics B, et al. Novel real-time PCR assay for detection of Helicobacter pylori infection and simultaneous clarithromycin susceptibility testing of stool and biopsy specimens. J Clin Microbiol 2004;42:4512-4518. doi:10.1128/ JCM.42.10.4512-4518.2004

5. Can F, Karahan C, Dolapci I, Demirbilek M, Tekeli A, Arslan H. Urease activity and urea gene sequencing of coccoid forms of Helicobacter pylori induced by different factors. Curr Microbiol 2008;56:150-155 doi:10.1007/s00284-007-9047-y

6. Ernst PB, Peura DA, Crowe SE. The translation of Helicobacter pylori basic research to patient care. Gastroenterology 2006;130:188-206 doi:10.1053/j.gastro.2005.06.032

7. Gisbert JP, Calvet X, Bermejo F, et al. III Conferencia Española de Consenso sobre la infección por Helycobacter pylori. Gastroenterol Hepatol 2013;36:340-374. doi:10.1016/j.gastrohep.2013.01.011
8. Malfertheiner P, Megraud F, O'Morain CA, et al. Management of Helicobacter pylori infection - the Maastricht V/Florence Consensus Report. Gut 2017;66:6-30. doi:10.1136/gutjnl-2016-312288

9. Mentis A, Lehours P, Mégraud F. Epidemiology and Diagnosis of Helicobacter pylori infection. Helicobacter 2015;20 Suppl 1:1-7. doi:10.1111/hel.12250

10. Lopes AI, Vale FF, Oleastro M. Helicobacter pylori infection - recent developments in diagnosis. World J Gastroenterol 2014;20:9299-9313.

11. Patel SK, Pratap CB, Jain AK, Gulati AK, Nath G. Diagnosis of Helicobacter pylori: what should be the gold standard? World I Gastroenterol 2014;20:12847-12859. doi:10.3748/wjg.v20.i36.12847

12. Parihar V, Holleran G, Hall B, Brennan D, Crotty P, McNamara D. A combined antral and corpus rapid urease testing protocol can increase diagnostic accuracy despite a low prevalence of Helicobacter pylori infection in patients undergoing routine gastroscopy. United European Gastroenterol J 2015;3:432-436. doi:10.1177/2050640615573374

13. Uotani T, Graham DY. Diagnosis of Helicobacter pylori using the rapid urease test. Ann Transl Med 2015;3:9. doi:10.3978/j.issn.23055839.2014.12.04

14. Calvet X, Sánchez-Delgado J, Montserrat A, et al. Accuracy of Diagnostic Tests for Helicobacter pylori: A Reappraisal. Clin Infect Dis 2009;48:1385-1391. doi:10.1086/598198

15. Zhou L, Zhao F, Hu B, et al. A creative Helicobacter pylori diagnosis scheme based on multiple genetic analysis system: qualification and quantitation. Helicobacter 2015;20:343-652. doi:10.1111/hel.12206

16. Ramírez-Lázaro MJ, Lario S, Casalots A, et al. Real-Time PCR Improves Helicobacter pylori Detection in Patients with Peptic Ulcer Bleeding. PLoS One 2011;6:e20009. doi:10.1371/journal.pone.0020009

17. Siavoshi F, Sainee P, Khalili-Samani S, et al. Evaluation of methods for H. pylori detection in PPI consumption using culture, rapid urease test and smear examination. Ann Transl Med 2015;3:11. doi:10.3978/j. issn.2305-5839.2014.11.16

18. El-Zimaity H, Serra S, Szentgyorgyi E, Vajpeyi R, Samani A. Gastric biopsies: the gap between evidence-based medicine and daily practice in the management of gastric Helicobacter pylori infection. Can J Gastroenterol 2013;27:e25-e30. doi:10.1155/2013/897423

19. Shirin D, Matalon S, Avidan B, Broide E, Shirin H. Real-world Helicobacter pylori diagnosis in patients referred for esophagoduodenoscopy: The gap between guidelines and clinical practice. United European Gastroenterol J 2016;4:762-769. doi:10.1177/2050640615626052

20. Saniee P, Shahreza S, Siavoshi F. Negative Effect of Proton-pump Inhibitors (PPIs) on Helicobacter pylori Growth, Morphology, and Urease Test and Recovery after PPI Removal--An In vitro Study. Helicobacter 2016;21:143-152. doi:10.1111/hel.12246

21. Nasser SC, Slim M, Nassif JG, Nasser SM. Influence of proton pump inhibitors on gastritis diagnosis and pathologic gastric changes. World J Gastroenterol 2015;21:4599-4606. doi:10.3748/wjg.v21.i15.4599

22. Malfertheiner P. Diagnostic methods for H. pylori infection: choices, opportunities and pitfalls. United European Gastroenterol J 2015;3:429431. doi:10.1177/2050640615600968

23. Yakoob J, Jafri W, Abid S, et al. Role of rapid urease test and histopathology in the diagnosis of Helicobacter pylori infection in a developing country. BMC Gastroenterol 2005;5:38. doi:10.1186/1471230X-5-38

24. McNicholl AG, Ducons J, Barrio J, et al. Accuracy of the Ultra-Rapid Urease Test for diagnosis of Helicobacter pylori infection. Gastroenterol Hepatol 2017;40:651-657. doi:10.1016/j.gastrohep.2017.07.007

25. Vaira D, Vakil N, Gatta L, et al. Accuracy of a new ultrafast rapid urease test to diagnose Helicobacter pylori infection in 1000 consecutive 
dyspeptic patients. Aliment Pharmacol Ther 2010;31:331-338. doi:10.1111/j.1365-2036.2009.04196.x

26. Bazin T, Nchare Mfondi A, Julie C, Émile JF, Raymond J, Lamarque D. Contribution of genetic amplification by PCR for the diagnosis of Helicobacter pylori infection in patients receiving proton pump inhibitors. United European Gastroenterol J 2018;6:1267-1273. doi:10.1177/2050640618787055

27. Yakoob J, Jafri W, Abbas Z, Abid S, Islam M, Ahmed Z. The diagnostic yield of various tests for Helicobacter pylori infection in patients on acid-reducing drugs. Dig Dis Sci 2008;53:95-100. doi:10.1007/s10620-007-9828-y

28. Atkinson NSS, Braden B. Helicobacter Pylori Infection: Diagnostic Strategies in Primary Diagnosis and After Therapy. Dig Dis Sci 2016;61:19-24. doi:10.1007/s10620-015-3877-4
29. Calvet X. Diagnosis of Helicobacter pylori Infection in the Proton Pump Inhibitor Era. Gastroenterol Clin North Am 2015;44:507-518. doi:10.1016/j.gtc.2015.05.001

30. Chen T, Meng X, Zhang H, Tsang RW, Tsang TK. Comparing Multiplex PCR and Rapid Urease Test in the Detection of H. pylori in Patients on Proton Pump Inhibitors. Gastroenterol Res Pract 2012;2012:898276. doi:10.1155/2012/898276

31. Cosme A, Torrente Iranzo S, Montes Ros M, et al. Helicobacter pylori antimicrobial resistance during a 5-year period (2013-2017) in northern Spain and its relationship with the eradication therapies. Helicobacter 2019;24:e12557. doi:10.1111/hel.12557 